Full length article

\title{
"Those edibles hit hard": Exploration of Twitter data on cannabis edibles in the U.S
}

\author{
Francois R. Lamy ${ }^{\mathrm{a}, \mathrm{b}, *}$, Raminta Daniulaityte ${ }^{\mathrm{a}}$, Amit Sheth ${ }^{\mathrm{b}}$, Ramzi W. Nahhas ${ }^{\mathrm{c}, \mathrm{f}}$, \\ Silvia S. Martins ${ }^{\mathrm{d}}$, Edward W. Boyer ${ }^{\mathrm{e}}$, Robert G. Carlson ${ }^{\mathrm{a}}$ \\ a Center for Interventions, Treatment, and Addictions Research (CITAR), Department of Community Health, Wright State University Boonshoft School of \\ Medicine, 3171 Research Blvd., Suite 124, Dayton, OH 45420-4006, United States \\ ${ }^{\mathrm{b}}$ Ohio Center of Excellence in Knowledge-enabled Computing (Kno.e.sis), Department of Computer Science and Engineering, Wright State University, \\ Dayton, $\mathrm{OH}$, United States \\ ${ }^{c}$ Center for Global Health, Department of Community Health, Wright State University Boonshoft School of Medicine, Dayton, OH, United States \\ d Department of Epidemiology, Columbia University Mailman School of Public Health, New York, NY, United States \\ e Department of Emergency Medicine, University of Massachusetts Medical School, Worcester, MA, United States \\ ${ }^{\mathrm{f}}$ Department of Psychiatry, Wright State University Boonshoft School of Medicine, Dayton, OH, United States
}

\section{A R T I C L E I N F O}

\section{Article history:}

Received 10 November 2015

Received in revised form 20 April 2016

Accepted 22 April 2016

Available online 26 April 2016

\section{Keywords:}

Twitter

Cannabis edibles

Social media

Cannabis

Cannabis legislation

\begin{abstract}
A B S T R A C T
Aims: Several states in the U.S. have legalized cannabis for recreational or medical uses. In this context, cannabis edibles have drawn considerable attention after adverse effects were reported. This paper investigates Twitter users' perceptions concerning edibles and evaluates the association edibles-related tweeting activity and local cannabis legislation.

Methods: Tweets were collected between May 1 and July 31, 2015, using Twitter API and filtered through the eDrugTrends/Twitris platform. A random sample of geolocated tweets was manually coded to evaluate Twitter users' perceptions regarding edibles. Raw state proportions of Twitter users mentioning edibles were ajusted relative to the total number of Twitter users per state. Differences in adjusted proportions of Twitter users mentioning edibles between states with different cannabis legislation status were assesed via a permutation test.

Results: We collected 100,182 tweets mentioning cannabis edibles with $26.9 \%(n=26,975)$ containing state-level geolocation. Adjusted percentages of geolocated Twitter users posting about edibles were significantly greater in states that allow recreational and/or medical use of cannabis. The differences were statistically significant. Overall, cannabis edibles were generally positively perceived among Twitter users despite some negative tweets expressing the unreliability of edible consumption linked to variability in effect intensity and duration.

Conclusion: Our findings suggest that Twitter data analysis is an important tool for epidemiological monitoring of emerging drug use practices and trends. Results tend to indicate greater tweeting activity about cannabis edibles in states where medical THC and/or recreational use are legal. Although the majority of tweets conveyed positive attitudes about cannabis edibles, analysis of experiences expressed in negative tweets confirms the potential adverse effects of edibles and calls for educating edibles-naïve users, improving edibles labeling, and testing their THC content.
\end{abstract}

(C) 2016 Elsevier Ireland Ltd. All rights reserved.

\section{Introduction}

In recent years, the legal status of cannabis has evolved rapidly in the U.S. with 23 states and the District of Columbia autho-

\footnotetext{
* Corresponding author at: Center for Interventions, Treatment, and Addiction Research (CITAR), 3171 Research Blvd, 124A, Kettering, OH 45420-4006, United States.

E-mail addresses: francois.lamy@wright.edu, flamy1978@gmail.com (F.R. Lamy).
}

rizing medical cannabis, including five that permit recreational use of cannabis (National Conference of State Legislatures, 2015). With these changes, new types of cannabis products have emerged (e.g., marijuana concentrates, THC syrup, e-cannabis), while others are becoming more readily available, such as leaf marijuana and cannabis edibles. The latter products can take several forms, most generally baked goods (e.g., cookies, brownies, muffins), candies (e.g., caramels, chocolates, hard candies, gums, "Rice Krispies," lollipops), or infusions. With increased availability, these products have also raised health concerns after reports of acute intoxications 
(Bui et al., 2015; Wang et al., 2013), suicide attempts (HancockAllen et al., 2015), and other dangers posed by potential access by children, particularly in cases where packaging mimics candies and other marketed products (Wang et al., 2013). Despite the growing media reports about acute intoxications linked to edibles (CBS, 2014; CPR, 2015; Ghosh et al., 2015), epidemiologic surveys focusing on cannabis edibles remain scarce, mainly because classic surveillance systems do not specifically monitor the use of cannabis edibles apart from cannabis consumption (SAMHSA, 2014). Overall, little is known about the patterns of use and users' perceptions of edible cannabis products.

In the last few years, there has been an increased interest in the use of large-scale web-based data as a timely source of information on evolving public health issues and trends (Butler et al., 2007; Daniulaityte et al., 2015; Eysenbach, 2011; Lazer et al., 2009; Miller and Sonderlund, 2010). Although there are a large variety of social media platforms, Twitter is of particular interest for public health research, because Twitter data are publicly accessible and reflect casual, unedited disclosures and communications from large numbers of people. Twitter is a micro-blogging platform enabling people to communicate via short posts (140 characters). With an average of 500 million tweets posted every day worldwide, Twitter represents a large and constantly updated source of information (Twitter, 2015). In the U.S., 19\% of the entire population is comprised of active Twitter users, with $30 \%$ of these tweeting daily. Furthermore, the U.S. Twitter population is young (32\% aged between 18 and 29 years), which makes it even more suitable for drug abuse surveillance since young adults display the highest rates of cannabis and most other drug use (SAMHSA, 2014). Twitter population in the U.S. is also ethnically diverse (e.g., $28 \%$ African-American, 28\% Hispanic) and educated (50\% with at least some college education; PewResearch, 2015). Because of the volume of data generated by Twitter users and also availability of geographic information, analysis of tweets can provide valuable population level metrics.

Several researchers have already illustrated the usefulness of Twitter in the field of drug abuse research. Hanson et al. (2013) analyzed geographic patterns of GPS-enabled tweets and found greater prevalence of Adderall-related tweets in the northeastern portion of the U.S. and also in some of the southern states. Cavazos-Rehg et al., (2015b) found that the prevalence of pro-alcohol tweets was significantly greater than anti-alcohol tweets and Twitter users' opinions of marijuana were predominantly positive (Cavazos-Rehg et al., 2015a). West et al. (2012) found that alcohol-related tweets were predominatly sent during night time and over weekends, with higher rates during historically typical drinking time periods (e.g., Christmas, New Years Eve). Another study demonstrated that marijuana concentrate-related tweeting activity was significantly greater in U.S. states that allow recreational and/or medical cannabis use (Daniulaityte et al., 2015). By analyzing Twitter data, the present paper aims to: 1) compare tweeting activity related to cannabis edibles across U.S. states with different marijuana legalization policies, and 2) explore the content of tweets related to cannabis edibles.

\section{Methods}

\subsection{Data collection}

Tweets were collected using Twitter's streaming Application Programming Interface (API) that provides free access to $1 \%$ of all tweets. The Twitter data filtering and aggregation framework was available through the eDrugTrends platform (eDrugTrends, 2015). This system is the result of interdisciplinary collaboration between researchers in the Center for Interventions, Treatment and
Addiction Research (CITAR) and computer scientists Center Excellence in Knowledge Enabled Computing (Kno.e.sis Center). It is an innovative social media analysis tool that has been developed for semi-automated processing and visualization of thematic, spatiotemporal, and social network dimensions of Twitter and Web forum data on cannabis and synthetic cannabinoid use. Development of the eDrugTrends platform was based on previous research and infrastructure including Twitris, for analysis of Twitter data (http:// knoesis.or/amit/media) (Chen et al., 2012; Jadhav et al., 2010; Nagarajan et al., 2009; Sheth, 2009), and PREDOSE, for analysis of web forum data (Cameron et al., 2013; Daniulaityte et al., 2013).

For this study, we extracted Twitter data from May 1st, 2015 to July 31st, 2015. To collect relevant tweets, we created a list of 38 search terms based on expressions related to cannabis edibles. To avoid any confusion with non-cannabis products, cannabis terms (e.g., "weed", "space", "marijuana" or "pot") were combined with cooking/food terms (e.g., "brownie", "cookies" or "cakes") to create search terms such as "pot cookie", "space cake" or "weed brownie". To reduce "noise" (i.e., irrelevant tweets) during data collection, eDrugTrends offers the ability to create a "blacklist" of expressions generating irrelevant tweets. Six expressions were blacklisted ("wild edibles", "grow edibles", “incredible edibles", "hydroponic edibles", "grew edibles", and "forage edibles") to increase the precision of our data collection.

The Wright State University Institutional Review Board (IRB) determined that this research met the requirements for Human Subjects Research exemption 4 due to the public nature of the collected data. We slightly modified tweets used as examples to ensure the anonymity of Twitter users who had posted them.

\subsection{Data analysis to identify regional differences}

Geolocation information of tweets was processed by the eDrugTrends platform. Since the percentage of Twitter users enabling their geolocation features is rather low (Burton et al., 2012), a module was developed in eDrugTrends to enhance the rate of geolocation identification. This module processes information indicated in the user profile available for public viewing (including "standard" names such as "Columbus, $\mathrm{OH}$ " and slang terminology such as "Big Apple"). If the user profile location contains such information, eDrugTrends performs a reverse geolocation query using Google to obtain geo-coordinated data. The time zone of the Google response is then checked against the time zone of the tweet to insure the accuracy of the extracted geolocation information.

Tweets that contained geolocation information indicating a state in the U.S. were extracted for further analysis. To compare the prevalence of edibles-related tweets, we adjusted for the different levels of tweeting activity in each state. Similar to prior studies (Jashinsky et al., 2014; West et al., 2012), to calculate adjusted percentages, we first generated a "general" sample of tweets representing tweeting activity in each state. This sample consisted of the default random sample of $1 \%$ of all tweets provided by the Twitter API. A total of 1,805,277 tweets were collected during an 8-day period, with 209,837 (11.6\%) of these inside the U.S. with state-level geolocation information. These geolocated tweets were tweeted by a total of 161,860 unique users. Both our cannabis-edibles related tweets and general sample tweets were processed using the same method to extract geolocation information. To assess the adequacy of the general sample of tweets used in this study, we compared it to another general sample of tweets used in a previously conducted study (Chen et al., 2014) and also to U.S. Census population statistics. We found a near perfect correlation between the numbers of tweets per state in our sample and the previously generated tweet sample, as well as state population statistics (Pearson correlations were 0.98 and 0.99 , respectively). 
Adjusted state-specific percentages of edibles-related tweets were calculated in the following way: 1) Raw state-level proportions of unique Twitter users tweeting about cannabis edibles were calculated; 2) Adjusted percentages were calculated using general sample rates to account for different number of Twitter users in each state. In particular, for each state, we computed the ratio of the proportion of unique Twitter users tweeting about cannabis edibles within a particular state to the proportion of general sample Twitter users; 3 ) These ratios were then rescaled by dividing each by the sum of ratios across states and multiplying by 100 , resulting in adjusted state-specific percentages of Twitter users posting about edibles. Whereas comparisons between raw proportions are confounded by population size, the adjusted proportions are comparable. Similar approaches to normalizing Twitter data were used by previous studies (Jashinsky et al., 2014; West et al., 2012).

To assess the relationship between cannabis legislation and edibles-related tweets, we differentiated states based on three types of legislation: (1) states where recreational and medical cannabis use is legal; (2) states where medical marijuana is permitted (excluding states with limited access marijuana product laws that allow medical use of high $\mathrm{CBD} /$ low THC cannabis products) and; (3) remaining states where both recreational and full access medical use of cannabis are illegal (National Conference of State Legislatures, 2015). Table 1 lists the different states according to their legislation status.

Differences between legal status groups in average adjusted percentage of Twitter users mentioning edibles were evaluated by performing a permutation test with 10,000 replications using $R$ (R Core Team, 2014). We tested the null hypothesis of no difference between each pair of legal status groups, adjusting for multiple testing using the Hommel method (Hommel, 1989).

\subsection{Content analysis of tweets}

The large volume of data extracted from social media does not allow a complete manual coding, and selection of random posts is a common strategy for content analysis of social media (Butler et al., 2007; McNaughton et al., 2014). Hence, we generated a random sample of 3000 tweets geolocated in the U.S. (one thousand per month of data collection, which represents $11.1 \%$ of the complete data set, $n=26,975$ ) for content analysis. The sample was coded manually using the qualitative analysis software QDA Miner (Provalis Research, 2011). First, 400 tweets were openly coded to explore emerging issues and themes and develop a coding scheme. Then, we coded the whole sample of tweets based on this coding scheme. The coding scheme included the following codes:

1) All tweets that were not directly related to cannabis edibles were coded Irrelevant.

2) We differentiated three types of tweet sources: Media tweets encompassed all news-related tweets, including retweets of news stories, political debates, scientific study results, and other reports (e.g., "Canadian Supreme Court Rules in Favour of Cannabis Edibles Reports Lexaria" or "RT @WeedFeed: A Complete Guide to \#Marijuana Edibles to answer your consumption questions"); Retail tweets originating from edible shops or cannabis dispensaries (e.g., "Sunday Funday!! All Edibles, Glass and accessories are 20\% off!! All flowers are 5G/40, 105/half \& 200/oz"); and Users tweets posted by Twitter users giving their opinions on edibles, describing experiences of consumption, an intention of use, or asking questions regarding cannabis edibles (e.g., "I need more edibles in my life" or "Edibles are for people who cant smoke").

3) For sentiments expressed in the tweets, we coded Users' tweets according to three categories. Tweets were labeled Negative when expressing a negative attitude toward edibles or unde- sired/detrimental effects (e.g., "These edibles have me so focused on not dying" or "Edibles are the demon"). Conversely, tweets denoting intention to use edibles, positive representation, intention to sell/give away or humorous tweets were coded Positive (e.g., "Edibles are love" or "Been trying to light this pot cookie for thirty minutes"). Finally, tweets coded Neutral included messages that do not contain enough information to be coded as positive or negative and tweets that do not express an opinon or sentiment about edibles (e.g., "Edibles" or "The marijuana cookie kid is one of my neighbors").

4) After manual coding was complete, we conducted computerbased word frequency analysis using WordStat software (Provalis Research, 2012). The key goal was to examine differences in the most common words appearing in tweets classified by source and sentiment. Identification of distinct linguistic patterns will eventually help create more automated content analysis tools. First, we filtered words that were used as keywords to obtain the relevant tweet sample (such as "edibles"; "marijuana"; "pot," and "cannabis"). Next; we eliminated commonly used "stop words," such as "to"; "the"; "and" or "all". Finally; we also grouped words that contained the same stem into the same category. For example; the words "smoked"; "smoking"; "smokes"; and "smoke" were encompassed in the term "smoke".

5) As a final stage, Tweets with positive or negative opinion were further explored using qualitative open coding to understand the key elements distinguishing positive and negative opinions about cannabis edibles.

\subsection{Reliability assessment of content analysis coding}

A random sub-sample of 300 tweets was selected for reliability assessment of the key elements of the coding scheme (Table 2). After clarifying and pre-testing coding rules, the reliability subsample was independently coded by two coders (the first and second authors) using QDA Miner. QDA Miner was then used to calculate (1) percentage agreement; and (2) Krippendorff's Alpha, which takes into account chance correction when calculating intercoder agreement (Krippendorff, 2012). Krippendorff's Alpha of $0.60-0.80$ indicates moderate and above 0.8 indicates substantial agreement (Bernard and Ryan, 2009).

\section{Results}

\subsection{Regional differences in tweeting activity related to cannabis edibles by legal status}

Over a three month period (May 1-July 31, 2015), 100,182 tweets related to cannabis edibles were collected, and 26,975 ( $26.9 \%$ of our sample) contained U.S. state-level geolocation information. These geolocated tweets were posted by 17,265 unique Twitter users. Raw counts of Twitter users tweeting about marijuana edibles were the highest in California (23.7\% of tweets across the U.S.), Texas (9.3\%), and New York (7.8\%). However, after adjusting for the different levels of unique Twitter users for each state based on the general sample, Colorado (5.9\%), Oregon (4.9\%), and Washington (4.0\%) had the highest adjusted proportions of unique Twitter users posting about edibles (Fig. 1).

The differences between states with different cannabis-related legal status in average adjusted proportion of Twitter users tweeting about cannabis edibles were statistically significant. The average adjusted proportion of unique Twitter users posting about cannabis edibles for Status 1 states (recreational and medical use legal) was $4.9 \%$, for Status 2 states (full access medical use permitted) $2.2 \%$, and for Status 3 states (illegal) $1.5 \%$. After adjusting for 
Table 1

States repartition depending on their cannabis legislation.

\begin{tabular}{|c|c|}
\hline Status (Code) & States \\
\hline Legal/Recreational and Medical THC (1) & AK, CO, DC, OR, WA \\
\hline Medical THC (2) & AZ, CA, CT, HI, IL, MA, MD, ME, MI, MN, MT, NH, NJ, NM, NV, NY, RI, VT \\
\hline Medical CBD/Decriminalized/Felony (3) & AL, AR, DE, FL, GA, IA, ID, IN, KS, KY, MO, MS, NC, OK, LA, NE, ND, OH, PA, SC, SD, TN, TX, UT, VI, WI WV, WY \\
\hline
\end{tabular}

Table 2

Content analysis and coding reliability assessment of tweets related to marijuana edibles.

\begin{tabular}{|c|c|c|c|c|}
\hline \multicolumn{3}{|c|}{ Content analysis, $\mathrm{N}=3000$ tweets } & \multicolumn{2}{|c|}{ Coding reliability assessment, $\mathrm{N}=300$ tweets } \\
\hline Code/Theme & Number & Percentage & Percentage agreement & K Alpha score \\
\hline Unrelated tweets & 357 & $11.9 \%$ & $94.3 \%$ & 0.68 \\
\hline Media related & 744 & $24.8 \%$ & $93.3 \%$ & 0.83 \\
\hline Retail related & 246 & $8.2 \%$ & $95.3 \%$ & 0.64 \\
\hline User generated & 1653 & $55.1 \%$ & $92.0 \%$ & 0.84 \\
\hline Positive & 939 & $56.8 \%$ & $86.0 \%$ & 0.68 \\
\hline Negative & 218 & $13.2 \%$ & $95.0 \%$ & 0.69 \\
\hline Neutral & 496 & $30.0 \%$ & $87.3 \%$ & 0.49 \\
\hline
\end{tabular}

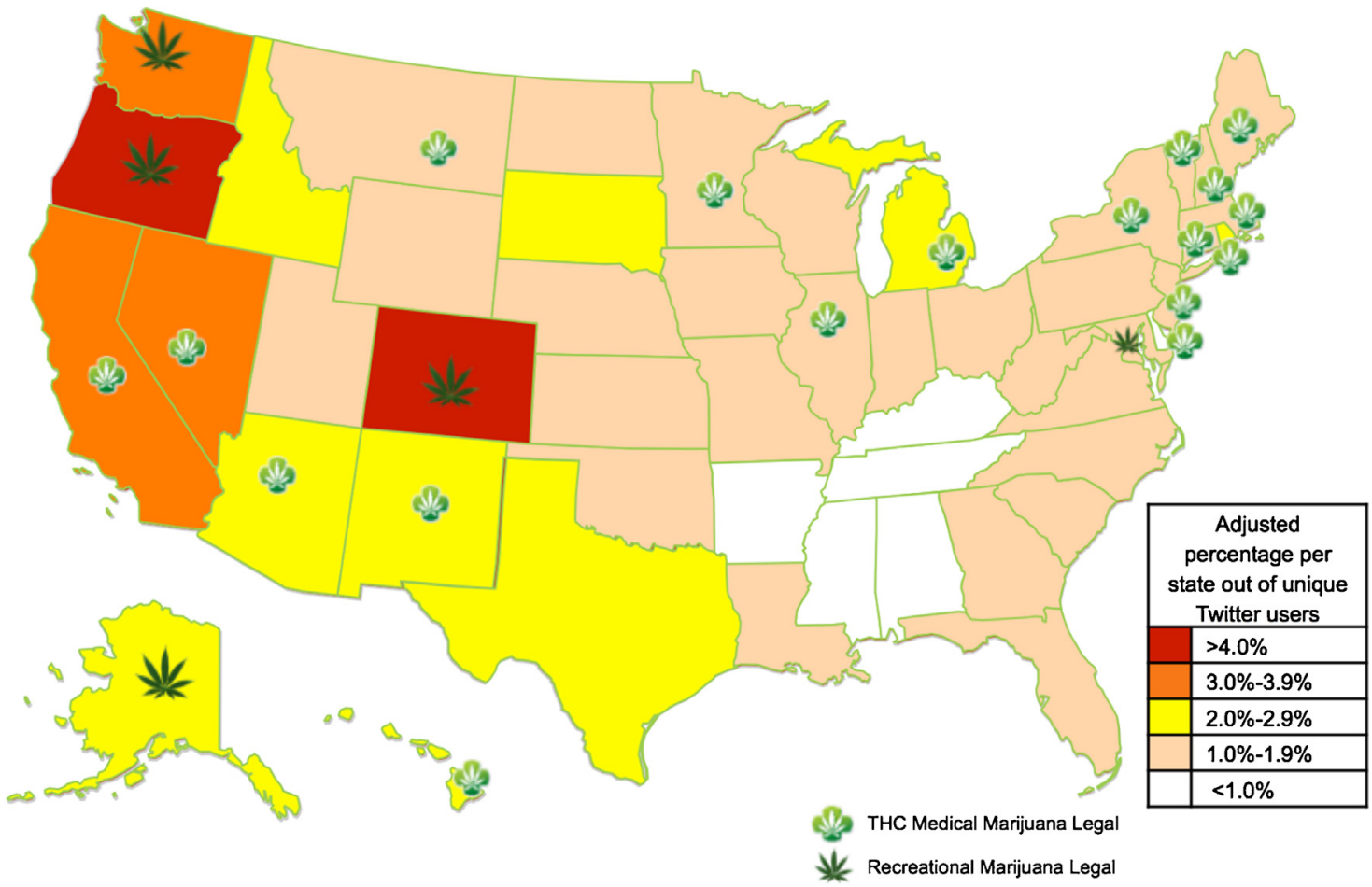

Fig. 1. U S. state-level repartition of edibles-posting Twitter users.

multiple comparisons, all three pairwise differences were significantly different from $0(\mathrm{p}<0.01)$.

Fig. 1 displays adjusted proportions of Twitter users tweeting about cannabis edibles by state. In addition to differences by state legal status, adjusted proportions of cannabis-related tweeting activity were greater in the Western part of the country, compared to full access medical cannabis states on the East Coast and Midwest. Our results also tend to indicate that states neighboring states with legislation authorizing recreational or medical THC have higher percentages of Twitter users posting about cannabis edibles (for example Idaho and South Dakota), despite conservative cannabis laws.

\subsection{Content analysis of tweets}

Results of the content analysis are displayed in Table 2. The searched terms used to collect Twitter data through the Twitris platform displayed $88.1 \%$ precision for the 3000 sample tweets. Among the 2599 relevant tweets, Users tweets were the most prevalent $(55.1 \%)$, while $24.8 \%$ were related to Media, and $8.2 \%$ were coded Retail (Table 2). The coding of the 1653 User tweets revealed that $56.8 \%$ were positive, $13.2 \%$ negative, and $30.0 \%$ neutral, confirming the popularity of cannabis edibles. Intercoder reliability asessment of the sub-sample of tweets revealed substantial or moderate agreement for most of the codes used in the analysis (Table 2).

Word frequency analysis, conducted using WordStat software (Provalis Research, 2012), identified the most frequent words appearing in the distinct categories of tweets. Table 3 presents the five most frequent words per code. As expected, the term "Eat" (encompassing the words "eat", "ate", "eats", and "eating") was the most frequent term found in our sample of edibles-related tweets. Word frequencies concerning the types of tweets showed disparities: Retail most frequent words concerned promotions and 
Table 3

Results from the word content analysis per type of tweet and sentiment codes.

\begin{tabular}{|c|c|c|c|c|}
\hline \multicolumn{3}{|l|}{ User Tweets } & \multirow[t]{2}{*}{ Media tweets } & \multirow[t]{2}{*}{ Retail tweets } \\
\hline Positive & Negative & Neutral & & \\
\hline Eat $(9.5 \%)$ & Eat (21.6\%) & Eat (10.7\%) & Eat (22.7\%) & Today $(9.8 \%)$ \\
\hline Make (9.1\%) & Don't (14.2\%) & Snoop (6.5\%) & Raid (20.8\%) & Free (7.7\%) \\
\hline $\operatorname{Lol}(4.4 \%)$ & $\operatorname{High}(9.6 \%)$ & Make (4.0\%) & Shop (12.6\%) & Monday (6.1\%) \\
\hline Good (3.8\%) & Time (9.6\%) & Time $(2.6 \%)$ & Cops $(11.6 \%)$ & Chocolate (5.3\%) \\
\hline Smoke (3.6\%) & Fuck (6.9\%) & Lol $(2.2 \%)$ & High (10.4\%) & THC $(4.1 \%)$ \\
\hline
\end{tabular}

products (e.g., "chocolate" or "free"); those concerning the Media code were related to edibles-related event (such as a police raid on a marijuana dispensary). User tweets that were coded as positive were more likely to contain words such as "good" or "lol", while negative terms such as "fuck" or "dont" were more prevalent in tweets coded Negative. These results indicate that identification of distinct linguistic patterns in tweets could help with automation of content analysis tasks in the future.

Results from the qualitative open coding show that the main element distinguishing positive and negative tweets appeared to be consumption outcomes. For example, negatively coded tweets, such as "That was not edibles. That was a square shaped piece of my personal hell" or "I couldn't stop moving, I went outside on the balcony and kept praying to God after I had two edibles" indicate the difficulties some users have predicting the intensity and duration of the high they experienced after consuming cannabis edibles. Inability to achieve normal daily tasks due to duration unpredictability (e.g., “Edibles are 'day wasters': you will do nothing after eating one" or "Edibles got me stuck to the couch") were also frequently associated with negatively coded tweets.

The difficulties for users to assess both duration and intensity of the high are augmented by the unreliability of THC dosage as indicated on packaging. Analysis of the negatively coded tweets reveals that this unreliability (e.g., "Edibles. Might be high. Might not be") concerns both over-effectiveness such as, "Edibles need to be less unpredictable: I had ONE peppermint and slept for $6 \mathrm{~h}$. I had plans damn it" and under-effectiveness such as, "The last three times I had edibles they didn't work so I probably won't waste my money" or "Yeah. It's been $3 \mathrm{~h}$ and still not really high at all. Crispy edibles are not working on me because of something? Dunno".

The most frequent desired effects linked to positive opinion are facilitation of sleep (e.g., "Im gonna sleep like a baby tonight, I ate some edibles." or "eating edibles before bed is always a fantastic idea"), and "get high" (e.g., "Cassy needs to make more edibles: it's like the very first time I got High but $3 x$ better"). It is also noteworthy that some positively coded tweets consider edibles as a healthier alternative to smoking cannabis joints or concentrates (e.g., "Im about just be on the edibles" my lungs fucked up" or "I would legit quit smoking, if edibles were available more often."). Given the frequent recommendation on use of edibles for relieving pain by consumer centric information sources (Patient's Care Collective, 2011; United Patients Group, 2015), surprisingly, the occurrence of positive tweets expressing the use of edibles to reduce or alleviate pain was marginally present with only 5 tweets among the 1653 User tweets in our sample. Illustrative tweets included: "Back is killing me like a mother! Pot is not strong enough for this pain. Gonna take some edibles later. Run is gonna kill me" and "U should make your own cannabutter, is a good way 2 manage your pain w(ith) edibles".

Data analysis also reveals mentions of home-made preparations of cannabis edibles in several tweets. Sample tweets included: "I start my Edibles this week. Projecting costs, I can still make profit and charge less!" or "First try at making edibles lets gooooo". In addition, several tweets indicated selling home-made edibles online, primarly from users located in authorized medical THC cannabis states (i.e. California, Michigan, Nevada, New York, Oregon), with, for example, "Homemade edibles on deck. good-ass sizes and potent as fuck. hmu!!! $10 \$$ a piece."

\section{Discussion}

Our study indicates greater cannabis-edibles related tweeting activity in states where medical THC and/or recreational cannabis use have been legalized, and most tweets generally expressed positive attitudes about cannabis edibles. In Colorado and Washington, cannabis edibles can be obtained legally from licensed retailers. In addition, in several states across the country, such as California, Oregon, Arizona and others, qualified patients can obtain cannabis edibles at medical marijuana dispensaries. The highest adjusted percentages of Twitter users tweeting about edibles were in Colorado, Oregon and Washington, states where recreational cannabis consumption is legal. Although the legal market of Oregon was not in operation at the time our Twitter data were collected, Oregon has one of the oldest medical marijuana programs in the country (National Conference of State Legislatures, 2015), and among the highest rates of marijuana use in the general population. Moreover, Fig. 1 reveals that a greater percentage of Twitter users tweeting about cannabis edibles are located in western states of the United States (such as California, Nevada, New Mexico, Oregon, or Washington), where medical cannabis programs were introduced earlier than in other parts of the country (National Conference of State Legislatures, 2015). Although epidemiological data on the prevalence of cannabis edibles use in the general population is lacking, one study conducted in Washington state a year before recreational cannabis use became legal found that almost $80 \%$ of cannabis users recruited for a web-based survey reported using cannabis edibles in the past year (Kilmer et al., 2013). Analysis of Twitter data on marijuana concentrates identified similar regional differences, with Oregon, Colorado and Washington showing the highest adjusted percentages of dabs-related tweets (Daniulaityte et al., 2015). As noted in other studies, high doses of THC can induce anxiety, panic attacks, cognitive impairments, and psychotic episodes (Di Forti et al., 2009; Hall and Degenhardt, 2015). The higher THC concentration of these two products, both marijuana concentrates and cannabis edibles, might lead to both short- (e.g., panic attacks, difficulty to focus, lack of memory, loss of attention) and long-term (e.g., cannabinoid hyperemesis syndrome, psychosis, schizophrenia) harmful consequences on a sub-population of cannabis users located in or visiting those states.

Twitter data suggest predominantly positive attitudes toward cannabis edibles with $56.8 \%$ of tweets expressing positive opinion, compared to $13.2 \%$ of tweets denoting negative attitudes or describing detrimental experiences. Positive tweets describe the quality of the "high" experienced and how cannabis edibles facilitate falling asleep. Our data also indicate that several Twitter users consider cannabis edibles as healthier compared to smoking. These results are consistent with findings of Murphy and colleagues (Murphy et al., 2015), who suggested that among older cannabis users in California, edibles are considered a healthier alternative. 
Despite the overall positive attitude about edibles, the analysis of negative tweets reveals that the unreliability of edibles' THC dosage and delayed effects were linked to over-consumption of cannabis edibles which, in turn, could lead to potential harmful consequences. These adverse consequences seem to be linked to two main causes: unrealiable THC content labelling and user's lack of practical knowledge concerning edibles. According to Vandrey et al. (2015), U.S. edible medical cannabis products display inaccurate ratios of THC and Cannabidiol (CBD). Of the 75 products tested, 23\% were underlabeled by the producers, while $60 \%$ were overlabeled, increasing the risk of acute intoxication and/or overconsumption (Vandrey et al., 2015). In addition, the pharmacokinetic difference between inhaling and ingesting cannabis (Grotenhermen, 2003) leads some users who usually smoke marijuana to consume extra dose(s) of edibles before the initial one kicks in which, in turn, increases the risk of overdose (Monte et al., 2015; Murphy et al., 2015). As such, the population of regular cannabis smokers who are uneducated about edible consumption are at considerable risk of overdose, as New York Times columnist Maureen Dowd (Dowd, 2014) brought to national attention. Our findings reinforce and support the call of legislators and scientists to improve the labelling of edibles and testing the reliability of edibles' THC dosage to reduce potential harms linked to overdosage as well as access by children (MacCoun and Mello, 2015). Furthermore, preventive harm-minimization efforts are needed to educate non-regular edibles users about consumption of these products (Global Drug Survey, 2015; The Cannabist, 2014).

This research has limitations, most of which are inherent to the field of Internet-based research. First, demographic characteristics of Twitter users were not available, and data collection was limited to tweets written in English. Second, individuals living in states with liberal cannabis policies might feel less restricted in publicly acknowledging their use and/or displaying their geolocation, which could partially affect regional differences in cannabis ediblesrelated tweeting activity. Furthermore, more work is required to increase the amount and accuracy of geolocated data, in particular for more-fine-grained analyses of regional trends. For example, Drezde et al. (2013) demonstrated that their automated geolocation information extraction approach achieved over 90\% accuracy in identifying the country of a tweet, but dropped to $54.5 \%$ when the geolocation accuracy was measured to a resolution of 25 miles (Drezde et al., 2013). Third, these findings reflect the perception of a portion of Twitter users describing an experience of consumption or expressing their opinion about edibles and are not necesseraly correlated with actual use. For example, some Twitter users might have a positive perception of edibles but not be active edibles users, while others may be actual users but may not be willing to tweet about a negative experience. More research with community-recruited samples of active users are needed to better undestand trends and perceptions identified in Twitter-based research. Finally, as illustrated by the intercoder reliability assessment, the brevity, ambiguity, lack of context and pervasive use of slang terminology, turns content analysis of tweets into a difficult task even for human coders. Future work will include training a sentiment analysis algorithm to automatically categorize sentiments regarding cannabis edibles and other drugs. This sentiment analysis algorithm will be integrated into the eDrugTrends platform to strenghten its analysis capacities and extend our analysis to the total volume of tweets collected.

To the best of our knowledge, this is the first study attempting to describe and analyze Twitter data on cannabis edibles. In the constantly evolving legal context surrounding cannabis products in the U.S., our research demonstrates that Twitter data analysis offers the possibility to retrieve timely information concerning trends of emerging cannabis product use, and provide new insights into the overall perceptions of Twitter users about cannabis prod- ucts. By providing an "insider" point-of-view, as well as timely and geolocated information, Twitter data could help policy makers to tackle and target specific aspects of a given phenomenon or trend. Furthermore, by using eDrugTrends as a tool to constantly collect Twitter data, we will be able to evaluate the performance and impact of newly implemented public health policies by monitoring changes in users experiences and opinions over time.

\section{Role of funding source}

This study was supported by the National Institute on Drug Abuse (NIDA), Grant No. R01 DA039454-01 (Daniulaityte, PI; Sheth, PI). The funding source had no further role in the study design, in the collection, analysis and interpretation of the data, in the writing of the report, or in the decision to submit the paper for publication.

\section{Contributors}

F. Lamy, R. Daniulaityte, A. Sheth, R. Carlson, R. Nahhas, S. Martins and E. Boyer designed the study. R. Nahhas helped with statistical analysis. F. Lamy reviewed the literature, wrote the first draft of the paper, extracted and coded the data. R. Daniulaityte participated to the manual coding and assessment review. A. Sheth also guided and supervised the development of eDrugTrend platform. All authors reviewed, commented, and edited the manuscript. All authors contributed to and have approved the final manuscript.

\section{Conflict of interest}

The authors declare that there are no conflict of interest.

\section{Acknowledgements}

The authors would like to acknowledge the role played by $\mathrm{G}$. Alan Smith and Sanjaya Wijeratne in designing and collecting data for the general sample described in the methodological section.

\section{References}

Bernard, H.R., Ryan, G.W., 2009. Analyzing Qualitative Data: Systematic Approaches. SAGE Publications, Thousand Oaks.

Bui, Q.M., Simpson, S., Nordstrom, K., 2015. Psychiatric and medical management of marijuana intoxication in the emergency department. West. J. Emerg. Med. $16,414$.

Burton, S.H., Tanner, K.W., Giraud-Carrier, C.G., West, J.H., Barnes, M.D., 2012. Right time, right place health communication on Twitter: value and accuracy of location information. J. Med. Internet Res. 14, e156.

Butler, S.F., Venuti, S.W., Benoit, C., Beaulaurier, R.L., Houle, B., Katz, N., 2007. Internet surveillance: content analysis and monitoring of product-specific internet prescription opioid abuse-related postings. Clin. J. Pain 23, 619-628.

CBS, 2014. Doctors Say E.R Visits Up Due To Marijuana Edibles, http://denver cbslocal.com/2014/04/24/edibles-the-main-culprit-when-it-comes-tomarijuana-hospital-visits/ (accessed on 08.03.15.).

CPR, 2015. Denver Emergency Room Doctor Seeing More Patients For Marijuana Edibles, http://www.cpr.org/news/story/denver-emergency-room-doctorseeing-more-patients-marijuana-edibles (accessed on 08.13.15.).

Cameron, D., Smith, G.A., Daniulaityte, R., Sheth, A.P., Dave, D., Chen, L., Anand, G., Carlson, R., Watkins, K.Z., Falck, R., 2013. PREDOSE: a semantic web platform for drug abuse epidemiology using social media. J. Biomed. Inform. 46, 985-997.

Cavazos-Rehg, P.A., Krauss, M., Fisher, S.L., Salyer, P., Grucza, R.A., Bierut, L.J., 2015a. Twitter chatter about marijuana. J. Adolesc. Health 56, 139-145.

Cavazos-Rehg, P.A., Krauss, M.J., Sowles, S.J., Bierut, L.J., 2015b. Hey everyone, I'm drunk: an evaluation of drinking-related twitter chatter. J. Stud. Alcohol Drugs 76, 635-643.

Chen, L., Wang, W., Sheth, A.P., 2012. Are Twitter Users Equal In Predicting Elections? A Study Of User Groups In Predicting 2012 US Republican Presidential Primaries Social Informatics. Springer, New York, pp. 379-392.

Chen, L., Weber, I., Okulicz-Kozaryn, A., 2014. US Religious Landscape On Twitter Social Informatics. Springer, New York, pp. 544-560.

Daniulaityte, R., Carlson, R.G., Falck, R., Cameron, D., Perera, S., Chen, L., Sheth, A., 2013. 'I just wanted to tell you that loperamide WILL WORK': a web-based study of extra-medical use of loperamide. Drug Alcohol Depend. 130, 241-244. 
Daniulaityte, R., Nahhas, R.W., Wijeratne, S., Carlson, R.G., Lamy, F.R., Martins, S.S., Boyer, E.W., Smith, G.A., Sheth, A., 2015. Time for dabs: analyzing Twitter data on marijuana concentrates across the US. Drug Alcohol Depend. 155, 307-311.

Di Forti, M., Morgan, C., Dazzan, P., Pariante, C., Mondelli, V., Marques, T.R., Handley, R., Luzi, S., Russo, M., Paparelli, A., Butt, A., Stilo, S.A., Wiffen, B., Powell, J., Murray, R.M., 2009. High-potency cannabis and the risk of psychosis. Br. J. Psychiatry 195, 488-491.

Dowd, M., 2014. Don't Harsh Our Mellow, Dude. The New York Times.

Drezde, M., Paul, M.J., Bergsma, S., Tran, H., 2013. Carmen: a twitter geolocation system with application to public health. AAAI Workshop on Expanding the Boundaries of Health Informatic Using AI (HIAI), 20-24

Eysenbach, G., 2011. Infodemiology and infoveillance tracking online health information and cyberbehavior for public health. Am. J. Prev. Med. 40 S154-S158.

Ghosh, T.S., Van Dyke, M., Maffey, A., Whitley, E., Erpelding, D., Wolk, L., 2015 Medical marijuana's public health lessons-implications for retail marijuana in Colorado. N. Engl. J. Med. 372, 991-993.

Global Drug Survey, 2015. The High-way Code: the Guide to Safer, More Enjoyable Drug Use, http://www.globaldrugsurvey.com/wp-content/uploads/2014/04/ High-Way-Code_Cannabis1.pdf (accessed on 08.16.15.).

Grotenhermen, F., 2003. Pharmacokinetics and pharmacodynamics of cannabinoids. Clin. Pharmacokinet. 42, 327-360.

Hall, W., Degenhardt, L., 2015. High potency cannabis. BMJ (Clin. Res. ed) 350 h1205.

Hancock-Allen, J.B., Barker, L., VanDyke, M., Holmes, D.B., 2015. Notes from the field: death following ingestion of an edible marijuana product-Colorado, March 2014. MMWR 64, 771-772.

Hanson, C.L., Burton, S.H., Giraud-Carrier, C., West, J.H., Barnes, M.D., Hansen, B. 2013. Tweaking and tweeting: exploring Twitter for nonmedical use of a psychostimulant drug (Adderall) among college students. J. Med. Internet Res. 15, e62.

Hommel, G., 1989. A comparison of two modified Bonferroni procedures. Biometrika 76, 624-625.

Jadhav, A.S., Purohit, H., Kapanipathi, P., Anantharam, P., Ranabahu, A.H., Nguyen, V., Mendes, P.N., Smith, A.G., Cooney, M., Sheth, A.P., 2010. Twitris 2.0: Semantically Empowered System for Understanding Perceptions from Social Data. http://corescholar.libraries.wright.edu/knoesis/252.

Jashinsky, J., Burton, S.H., Hanson, C.L., West, J., Giraud-Carrier, C., Barnes, M.D., Argyle, T., 2014. Tracking suicide risk factors through twitter in the US. Crisis 35, 51-59.

Kilmer, B., Caulkins, J.P., Midgette, G., Dahlkemper, L., MacCoun, R.J., Liccardo, P.R. 2013. Before the Grand Opening: Measuring Washington State's Marijuana Market in the Last Year Before Legalized Commercial Sales. Rand Corporation, New York.

Krippendorff, K., 2012. Content Analysis: An Introduction To Its Methodology. Sage, Thousand Oaks.

Lazer, D., Pentland, A.S., Adamic, L., Aral, S., Barabasi, A.L., Brewer, D., Christakis, N., Contractor, N., Fowler, J., Gutmann, M., 2009. Life in the network: the coming age of computational social science. Science 323, 721

MacCoun, R.J., Mello, M.M., 2015. Half-baked-the retail promotion of marijuana edibles. N. Engl. J. Med. 372, 989-991.
McNaughton, E.C., Black, R.A., Weber, S.E., Butler, S.F., 2014. Assessing abuse potential of new analgesic medications following market release: an evaluation of internet discussion of tapentadol abuse. Pain Med. 16, 131-140.

Miller, P.G., Sonderlund, A.L., 2010. Using the internet to research hidden populations of illicit drug users: a review. Addiction (Abingdon England) 105, 1557-1567.

Monte, A.A., Zane, R.D., Heard, K.J., 2015. The implications of marijuana legalization in Colorado. JAMA 313, 241-242.

Murphy, F., Sales, P., Murphy, S., Averill, S., Lau, N., Sato, S.-O., 2015. Baby boomers and cannabis delivery systems. J. Drug Issues 45, 293-313.

Nagarajan, M., Gomadam, K., Sheth, A.P., Ranabahu, A., Mutharaju, R., Jadhav, A., 2009. Spatio-Temporal-Thematic Analysis Of Citizen Sensor Data: Challenges And Experiences. Springer, New York.

National Conference of State Legislatures, 2015. State Medical Marijuana Laws, http://www.ncsl.org/research/health/state-medical-marijuana-laws.aspx (accessed on 08.20.15.)

Patient's Care Collective, 2011. Beginner's Guide to Medical Cannabis-using Edibles, http://berkeleypatientscare.com/2011/05/18/beginners-guide-tomedical-cannabis-using-edibles/ (accessed on 11.05.15.).

PewResearch, 2015. Twitter Demographic, http://www.pewinternet.org/2015/08/ 19/mobile-messaging-and-social-media-2015/2015-08-19_social-mediaupdate_11/ (accessed on 12.02.16.).

Provalis Research 2011 QDA Miner version 4.0 User Manual Montreal, QC, Canada. Provalis Research, 2012 WordStat. Provalis Research, Montreal, QC Canada.

R Core Team, 2014. R: A Language and Environment for Statistical Computing. R Foundation for Statistical Computing, Vienna, Austria, ISBN 3-900051-07-0, 2012

S.A.M.H.S.A, 2014. Results from the 2013 National Survey on Drug Use and Health: Detailed Tables. Office of Applied Studies, Rockville, MD.

Sheth, A., 2009. Citizen sensing, social signals, and enriching human experience. IEEE Internet Comput. 13, 87.

The Cannabist, 2014. Get Educated About Edibles: Eight Tips for Getting Right Dose, http://www.thecannabist.co/2014/04/02/edibles-getting-the-rightdose-infused-marijuana-colorado/8543/ (accessed on 08.12.15.).

Twitter, 2015. About Company, https://about.twitter.com/company (accessed on Web Page 2015).

United Patients Group, 2015. Ways to Consume Medical Marijuana, http://www unitedpatientsgroup.com/resources/methods-of-consumption (accessed on 11.05.15.).

Vandrey, R., Raber, J.C., Raber, M.E., Douglass, B., Miller, C., Bonn-Miller, M.O., 2015 Cannabinoid dose and label accuracy in edible medical cannabis products. JAMA 313, 2491-2493.

Wang, G.S., Roosevelt, G., Heard, K., 2013. Pediatric marijuana exposures in a medical marijuana state. JAMA Pediatr. 167, 630-633.

West, J.H., Hall, P.C., Hanson, C.L., Prier, K., Giraud-Carrier, C., Neeley, E.S., Barnes, M.D., 2012. Temporal variability of problem drinking on Twitter. Open J. Prev. Med. 2.

eDrugTrends, 2015. Project Information, http://wiki.knoesis.org/index.php/ EDrugTrends (accessed on 11.05.15.) 